1991-02-01

\title{
Convex Bayes decision theory
}

Wynn C. Stirling

wynn_stirling@byu.edu

Darryl Morrell

Follow this and additional works at: https://scholarsarchive.byu.edu/facpub

Part of the Electrical and Computer Engineering Commons

\section{Original Publication Citation}

Stirling, W. C., and D. R. Morrell. "Convex Bayes Decision Theory." Systems, Man and Cybernetics, IEEE Transactions on 21.1

\section{BYU ScholarsArchive Citation}

Stirling, Wynn C. and Morrell, Darryl, "Convex Bayes decision theory" (1991). Faculty Publications. 724. https://scholarsarchive.byu.edu/facpub/724 accepted for inclusion in Faculty Publications by an authorized administrator of BYU ScholarsArchive. For more information, please contact ellen_amatangelo@byu.edu. 


\title{
Convex Bayes Decision Theory
}

\author{
Wynn C. Stirling, Member, IEEE, and Darryl R. Morrell, Member, IEEE
}

\begin{abstract}
The basic concepts of Levi's epistemic utility theory and credal convexity are presented. Epistemic utility, in addition to penalizing error as is done with traditional Bayesian decision methodology, permits a unit of informational value to be distributed among the hypotheses of a decision problem. Convex Bayes decision theory retains the conditioning structure of probability-based inference, but addresses many of the objections to Bayesian inference through relaxation of the requirement for numerically definite probabilities. The result is a decision methodology that stresses avoiding errors, and seeks decisions that are likely to be highly informative as well as true. By relaxing the mandatory requirement for unique decisions and point estimates in all cases, decision and estimation criteria are obtained that do not demand more than it is possible to obtain from the data, and permit a natura man-in-the-loop interface. Applications are provided to illustrate the theory.
\end{abstract}

\section{INTRODUCTION}

$\mathrm{D}$ ECISION THEORY is a problem of epistemology; i.e., how to deal with the dynamics of changing knowledge and belief. In particular, as data are made available to an agent (an agent may be a person, a committee, an institution, or any other entity that is capable of making decisions), a central goal of decision theory is to define and evaluate criteria for decision making that meet the demands for knowledge that are imposed by the users of an information system. A typical approach is to introduce a notion of optimality, and to seek the corresponding optimal decision rule. When employing such a strategy, however, the agent must be assured that the criterion used for optimality is above controversy, and that there is sufficient modeling information available to operate the decision rule reliably. Difficulties arise if the implementation of an optimal decision strategy requires the agent to impose arbitrary modeling assumptions on the information system, or if there is genuine unresolved conflict regarding the criterion or the modeling assumptions. In these situations, the agent may be very reluctant to consign the decision solely to an autonomous algorithm. Unfortunately, the structure of many classical decision techniques is such that it is difficult to inject human judgment into the decision-making process. This problem is particularly evident when the decision rule is designed to provide "the truth and nothing but the truth," i.e., to yield one and only one decision output which is represented to the agent as the true answer, and does not provide alternative possibilities for consideration.

Manuscript received April 6, 1990; revised July 28, 1990. This work was supported by ESL, Inc., Sunnyvale, CA.

W. C. Stirling is with the Department of Electrical and Computer Engineering, 459 CB, Brigham Young University, Provo, UT 84602.

D. R. Morrell is with the Department of Electrical Engineering, Arizona State University, Tempe, AZ 85236-15706.

IEEE Log Number 9039998.
This paper discusses decision making from a perspective that, although firmly based in probability theory, differs in some important aspects from the strict Bayesian doctrine. A key feature of this approach is that it makes an important shift in the goals of traditional Bayesian-based decision theory without abandoning the strengths of the theory. Rather than attempt to minimize the effects of error, we pursue a goal of seeking to avoid error. Clearly, error cannot be completely avoided if new knowledge is to be seriously pursued, but a hypothesis being considered for inclusion into the body of knowledge should be of sufficient value to warrant the risk of error in accepting it. This shift in epistemological emphasis has significant implications concerning the structure of the decision rules and the man-machine interface that it makes possible.

\section{Epistemology}

The goal of rational inquiry is to acquire new knowledge, but at the same time seek to avoid introducing error into the body of knowledge. We shall be concerned, in this paper, with probabilistic models of epistemology in the spirit of [1]-[3]. Probability theory is well-suited to deal with error, but its successful implementation depends upon the availability of sufficient detail regarding the structure and parameters of the relevant probability models to ensure that the goals of minimizing the effects of error can be realized. When possession of this knowledge is questioned, it is tempting to reject probability-based inference as a viable means of rational decision making.

Considerable effort has been devoted to the development of alternatives to Bayesian probabilistic information processing in an attempt to alleviate some of the problems that have been identified with the classical approach. For example, the Dempster-Shafer theory of evidential reasoning [4] and Zadeh's fuzzy set theory [5], have been represented as alternatives to classical Bayesian inference. These approaches may reduce or eliminate some of the problems of probability-based inference, but they tend to introduce problems of their own-see, for example, [6], [7]. Critics of the probabilistic method charge that it is not well-suited for dealing with ignorance, which, according to its root meaning, is lack of knowledge. They do not accept the traditional probabilistic practice of expressing ignorance through maximum entropy probability distributions (thus equating ignorance with uncertainty, or imprecision) and argue that it is often difficult or impossible to specify the numerically definite probability distributions required for strict Bayesian inference. Stephanou and Sage [8] provide an informative discussion of other drawbacks of probabilistic-based decision methodology, including arguments regarding the semantic correspondence of probabilistic theory to natural language, and discuss 
and evaluate some alternatives that have been proposed. In spite of its real or apparent shortcomings, however, Bayesian inference is so powerful and attractive that it is not rejected easily as a means of combining evidence to reach conclusions. Some authors, (e.g., [7]) argue that probability is the only sensible way to describe uncertainty. Regardless of whether that argument is accepted or rejected, the probabilistic approach will perhaps remain the standard with respect to which alternative methods are evaluated.

The act of making decisions involves conflict between the desire to obtain new knowledge by extracting information from data or evidence about the system or process under inquiry, and the desire to avoid error. Thus, any agent undertaking a decision problem has, as a proximate aim of his investigation, the goal of acquiring error-free knowledge. ${ }^{1}$ Any decision strategy adopted by the agent should reflect his value system regarding the benefit of acquiring information versus introducing error into his body of knowledge. He should expect to revise his beliefs by judging not just truth, but truth of informationally valuable hypotheses. He may be reluctant to reject important hypotheses simply on the basis of the probability of truth and error, and he may be indifferent to the truth or error of a hypothesis he regards as informationally unimportant.

We follow Levi's epistemology [1], [9]-[11] in the development of this paper, and begin by discussing two principles that are desirable for rational decision making. The first principle is one that regulates the evaluation of information regardless of its truth-value; the second principle is one that regulates the credibility of information regardless of its worth. Using Levi's terminology, we denote the first principle as epistemic utility, and the second as credal probability. These notions, coupled with the key concepts of valuational convexity and credal convexity, lead to a decision strategy that addresses many of the problems that are often encountered with classical Bayesian decision formulations. We present these concepts and explain their relevance in an engineering context. Applications are provided to illustrate the operation of the resulting decision rules and to compare them with classical approaches.

\section{EPISTEMic Utility}

Levi [1], [10]-[12], has outlined an epistemological approach to decision making that we find to be an interesting and compelling model of knowledge and belief dynamics for a single agent, denoted by the symbol $X$. We assume, at time $t$, that $X$ possesses, with some degree of awareness and commitment, an epistemic system, denoted by the triple $\left(\mathscr{K}_{X, t}, \mathscr{G}_{X, t}, \mathscr{B}_{X, t}\right)$, consisting of the following.

- A knowledge corpus, $\mathscr{K}_{X, t}$ that (a) is deductively closed; i.e., it contains all deductive inferences derived from sentences in $\mathscr{K}_{X, t}$; and (b) $\mathscr{K}_{X, t}$ contains all logical truths, all set-theoretical truths, and all mathematical truths that are expressible in $X$ 's language. Although it may not be possible to specify exhaustively the contents of $\mathscr{K}_{X, t}$ and it is not assumed that $X$ possesses superhuman abilities to store information and make computa-

${ }^{1}$ As the American humorist Josh Billings put it: "It ain't so much ignorance that ails mankind as it is knowing so much that ain't so." tions, we do assume that $X$ is rational and lives up to the commitments contained in $\mathscr{K}_{X, t}$ insofar as he is able.

- A system of informational valuation, $\mathscr{G}_{X, t}$, that reflects the worth or value of any decision to $X$. Any agent undertaking a possible revision to his corpus (e.g., a decision) will be strongly influenced by the system he uses to evaluate the importance of each hypothesis under consideration. He may be willing to incur high risk of error to adopt a very valuable hypothesis, but will most probably be reluctant to assume any appreciable risk to adopt a hypothesis to which he is essentially indifferent, even though it may very likely be true. We shall assume that the valuation system consists of a collection of utility functions, each element of which is an equivalence class (i.e., equivalent up to positive linear transformations) of utilities.

- A system of truth valuation, $\mathscr{B}_{X, t}$, that reflects $X$ 's assessment of belief in options being true. Candidates for the truth-valuation system include probability-based approaches as well as alternatives such as Zadeh's fuzzy set theory [13] and Shafer's evidential reasoning [4]. In this paper, we shall represent the truth-validation by a convex set of probability functions, each member of which is a serious possibility for being correct.

There are three ways in which an agent may wish to modify his corpus of knowledge. First, he may expand his corpus by adding new knowledge. Second, he may contract his corpus by removing some statement that he no longer believes is true. Third, he may revise his corpus by replacing a suspect statement in his corpus with a new statement; revision may be viewed as a sequence of contractions and expansions. In this paper, we will focus primarily on the application of decision theory for the expansion of knowledge. The agent's current knowledge corpus, $\mathscr{K}_{X, t}$, is his standard for serious possibility for improving his knowledge. That is, he must evaluate new hypotheses as serious candidates for inclusion into his corpus by means of what he currently knows.

For a specific inquiry under investigation by agent $X$, suppose there are finitely many hypotheses, or options, that may be considered. Let $U$ denote this set of possible answers and assume that $\mathscr{K}_{X, t}$ entails exactly one element of $U$, and that all elements of $U$ are consistent with $\mathscr{K}_{X, t} . U$ is said to be an ultimate partition for $X$. A potential answer for $X$ occurs if he is able to reject all and only members of a subset of $U$. Let $n$ denote the number of elements in $U$. Then there are $2^{n}$ potential answers for $X$, including $\varnothing$, the contradictory potential answer. This collection of potential answers forms a Boolean algebra, denoted $\mathscr{A}$, of elements of $U$. A potential answer is degenerate if no elements of $U$ are rejected, in which case $\mathscr{K}_{X, t}$ remains unchanged. If all elements of $U$ are rejected, the agent expands into a contradiction; thus, $X$ may not reject all members of $U$. If $X$ rejects all but one element of $U$, the surviving hypothesis is the strongest potential answer for $X$, and is added to his corpus. When more than one element of $U$ survives rejection, $X$ remains in suspense between the rival un-rejected alternatives.

The conflict between making decisions (i.e., acquiring knowledge) and avoiding error can be represented as a convex combination of two utility functions: 1) the utility of 
avoiding error, and 2) the utility of obtaining new information (in this development, we follow [10, ch. 7]).

For any $g \subset U$, we define the utility of $X$ accepting $g$ in the interest of avoiding error as $T(g ; l)$, with $l \in\{t, f\}$ denoting whether $g$ is true $(t)$ or false $(f)$, according to $\mathscr{K}_{X, t}$, where

$$
T(g ; l)=\left\{\begin{array}{ll}
1 & \text { if } l=t \\
0 & \text { if } l=f
\end{array} .\right.
$$

This utility function is equivalent to a uniform cost function in classical Bayesian decision problem formulations $[14$, ch. 2]). In addition to the cost of error, however, we also apportion a unit of informational value among the options. To assess the utility of new information, $X$ may assign an informational value to each $g \in U$. The potential answers may be partially ordered with respect to informational value as follows. Let $g_{1} \subset U$ and $g_{1} \subset U$ be potential answers. Then accepting $g_{1}$ involves rejecting $\sim g_{1}$, the complement of $g_{1}$; similarly for $g_{2}$. If $\sim g_{1} \subset \sim g_{2}$ but $\sim g_{1} \neq \sim g_{2}$, then $g_{1}$ is less valuable informationally than $g_{2}$, i.e., accepting $g_{2}$ rejects more elements of $U$ than does accepting $g_{1}$. Rejecting no elements of $U$ is the least valuable of all the potential answers. Rejecting all of them (and, hence, contradicting oneself) is most valuable informationally.

$X$ may represent the informational value of potential answers by assigning to elements of $U$ non-negative real values such that their sum is unity. The informational value of a potential answer is the sum of the values assigned each element of $U$ that is rejected according to the potential answer; i.e., if we enumerate $U=\left\{h_{1}, h_{2}, \cdots, h_{n}\right\}$, and let $M\left(h_{j}\right) \geqslant 0$ denote the value $X$ assigns to $h_{j}$, then $\sum_{j=1}^{n} M\left(h_{j}\right)$ $=1$. For any set $g \subset U$, we define

$$
M(g)=\sum_{h_{j} \in g} M\left(h_{j}\right) .
$$

Then $M(g)$ is $X$ 's informational value of rejecting $g$, i.e., of adding $\cup_{h_{j} \notin g} h_{j}$ to $\mathscr{K}_{X, t}$.

The function $M(\cdot)$ thus defined is a probability over $\mathscr{A}$, the Boolean algebra of elements of $U$. Levi terms this probability an information-determining probability. ${ }^{2}$ It is intended to regulate the evaluation of information regardless of its truth-value - it is a measure of the demand for information. Thus, if $X$ considers rejecting $g$, he is certain that he will obtain informational value worth $M(g)$. he does not know whether $g$ is true or false. The utility of accepting $g$ in the interest of acquiring new information regardless of its truth-value is, then, $C(g)=1-M(g)$.

Given the utility function, $T(g ; l)$, of avoiding error and the utility function, $C(g)$, of acquiring information, we may address the conflict that exists between these two goals by defining an epistemic utility function for acquiring error-free knowledge as a convex combination:

$$
u(g ; l)=\alpha T(g ; l)+(1-\alpha) C(g) .
$$

The quantity $\alpha$ represents the relative importance that $X$ attaches to avoiding error versus acquiring new information.

\footnotetext{
${ }^{2}$ It should be noted that, since $M$ is used primarily as a weighting function to rank the importance of options, it is not strictly required to be a probability, and is never used to calculate expectations. We will, however, find it advantageous to retain Levi's characterization of this function as a probability.
}

We must restrict $\frac{1}{2} \leqslant \alpha \leqslant 1$ to ensure that no erroneous answer is preferred to any correct answer. Note that this utility consists of a component that is conditioned on the error (i.e., $T(g ; l))$ and a component that is associated with unconditional value (i.e., $M(g)$ ). This structure contrasts with the standard Bayesian formulation for the multiple hypothesis problem, where a cost $C_{k j}$ is assessed if the $k$ th hypothesis is chosen when the $j$ th hypothesis is true. Thus, the standard Bayes cost structure measures only the cost of error, whereas the dual nature of epistemic utility permits both the cost of error and the informational value of the decisions to be specified independently. This utility function reduces to the uniform cost criterion when $\alpha=1$.

Since all utility functions that are related by a positive linear transformation are equivalent, we may. simplify this utility function by defining

$$
u^{a}(g ; l)=\frac{1}{\alpha} u(g ; l)-\frac{1-\alpha}{\alpha} .
$$

The resulting utility function for accepting $g$ in the interest of both avoiding error and acquiring new knowledge is

$$
u^{a}(g ; l)=\left\{\begin{array}{ll}
1-b M(g) & \text { if } l=t \\
-b M(g) & \text { if } l=f
\end{array},\right.
$$

where $b=(1-\alpha) / \alpha$.

\section{Credal Probability and Expected Utility}

Independently from establishing a utility to characterize the informational value of accepting or rejecting a given option, the agent must also establish a probabilistic measure of belief for the options that are available. Credal probability is probability formed on the basis of subjective judgment, represents the likelihood that an option is true, and is independent of any informational value or demand that might be associated with the option. In the Bayesian context, prior probabilities are credal probabilities reflecting the agent's $a$ priori beliefs concerning elements of the ultimate partition, conditional probabilities are credal probabilities reflecting the agent's beliefs concerning the interrelationships between the hypotheses and the data or evidence, and posterior probabilities are credal probabilities reflecting the agent's state of belief after the prior and conditional probabilities have been combined via Bayes rule. Whereas the information-determining probability is used to determine the utility of error-free knowledge, credal probability may be viewed as expectation-determining probability.

For a given ultimate partition $U$, let $Q(g)$ denote $X$ 's credal probability assignment to any element $g \in \mathscr{A}$, the Boolean algebra of elements in $U$. If $g$ bears positive credal probability, then $X$ evaluates the truth of $g$ as a serious possibility. For $g \in \mathscr{A}$, let

$$
\begin{aligned}
E_{Q} u_{a}(g ; l) & =[1-b M(g)] Q(g)-b M(g)[1-Q(g)] \\
& =Q(g)-b M(g),
\end{aligned}
$$

where $E_{Q}(\cdot)$ is the mathematical expectation. This quantity is the expected epistemic utility with respect to the probability $Q$. A well-accepted and rational strategy for an agent to adopt is to make decisions in a way that tends to maximize his expected utility. Thus, for any element $h_{i} \in U$, the strat- 
egy of accepting $h_{i}$ bears an expected utility of

$$
E_{Q} u_{a}\left(h_{i} ; l\right)=Q\left(h_{i}\right)-b M\left(h_{i}\right) .
$$

We may explain the terms in this equation as follows.

$M\left(h_{i}\right)$ represents the informational value of rejecting $h_{i}$. If $M\left(h_{i}\right)<M\left(h_{j}\right)$, then rejecting $h_{j}$ is informationally more valuable than rejecting $h_{i}$ or, equivalently, accepting $h_{i}$ is of more informational value than accepting $h_{j}$.

$Q\left(h_{i}\right)$ represents subjective probability that $h_{i}$ is true. If $Q\left(h_{i}\right)<Q\left(h_{j}\right)$, then $X$ is able to rank hypothesis $h_{j}$ as being more likely than hypothesis $h_{i}$.

$b \quad X$ 's index of boldness. Since $\frac{1}{2} \leqslant \alpha \leqslant 1$, we have $0 \leqslant b \leqslant 1$. If $b=1$, the relative importance of avoiding error is minimum. The closer $b$ is to unity, the less caution $X$ exercises that error will be introduced into his corpus; i.e., he is maximally bold. The closer $b$ is to zero, the lower the risk or error he will accept before being willing to expand his corpus. Classical Bayesian decision rules with uniform cost correspond to $b=0$.

Thus, the expected utility represents a tradeoff between the desire to add new knowledge to the corpus and the desire to avoid error. The choice of $b$ establishes the agent's threshold at which the demand for knowledge renders the risk or error worthwhile.

The agent $X$ may adopt any option in the Boolean algebra generated by the elements of the ultimate partition, $U$. This possibility expands $X$ 's possibilities; he is not constrained to select only the elementary events, $h_{i}$, but may choose any subset of them (such a choice means, in practice, that the agent is rejecting the complement of the accepted set of options). The option that maximizes expected utility of acceptance is the set $g^{*}=\left\{h_{i} \in U: Q\left(h_{i}\right)-b M\left(h_{i}\right) \geqslant 0\right\}$. Thus, the criterion of maximizing expected utility is equivalent to rejecting all options $h_{i}$ such that $Q\left(h_{i}\right)<b M\left(h_{i}\right)$. If there is only one element $h_{i *} \in U$ such that $Q\left(h_{i *}\right) \geqslant b M\left(h_{i *}\right)$, then there is a unique maximizing (with respect to expected utility) decision. If $g^{*}$ contains more than one element, then none of them may be rejected on the basis of expected utility. The essence of this decision-making strategy is that, rather than selecting the one "best" option, we reject only the "bad" options, leaving, as potential answers, all of the "good" options for further consideration-i.e., we place a higher priority on avoiding error rather than seeking the one option with smallest probability of error, which would be represented to the agent as the truth. This decision philosophy may be summarized as follows:

Levi's Rule of Expected Utility [1, p. 53]: Given a corpus $\mathscr{K}_{X, t}$, a finite ultimate partition $U$, an information-determining probability function $M$ defined over the Boolean algebra of elements of $U$, an expectation-determining probability function $Q$ defined over the same algebra, and an index of boldness $b$, the agent should reject all and only those elements of $h_{i} \in U$ satisfying $Q\left(h_{i}\right)<b M\left(h_{i}\right)$.

The test $Q\left(h_{i}\right)-b M\left(h_{i}\right) \geqslant 0$ is a likelihood ratio test involving two probabilities: (a) the information-determining probability, $M$, and (b) the credal or expectation-determining probability, $Q$. If we fail to reject all hypotheses $h_{i}$ for which $Q\left(h_{i}\right) \geqslant b M\left(h_{i}\right)$, we accept each hypothesis $h_{i}$ whose ratio of credal probability of being true to the informational value of rejecting it exceeds the boldness threshold, $b$.

Levi's rule of expected utility may be contrasted with other possible classical decision-making strategies. Conventional multiple hypothesis testing entails ranking the expected utilities of all the $h_{i}$ 's and selecting as optimal that option $h_{i}$ that has the highest ranking. This strategy is more bold than Levi's rule, and results in rejecting all but one hypothesis; even though there may be other hypotheses that have nearly as high expected utility, and may have lower utility if in error. Conventional maximin entails the calculation of the utilities of all options and selecting as optimal that option $h_{i}$ such that $\min _{l} u\left(h_{i}, l\right) \geqslant \max _{j \neq i} \min _{l} u\left(h_{j}, l\right)$, where $l \in\{t, f\}$. This strategy is less bold than Levi's and, though the resulting decision maximizes the minimum utility, it may commit the agent to an option that is extremely unlikely to occur.

Most traditional decision rules are based only upon assessments of truth or error. This criterion does seem to be the appropriate one in many contexts, such as statistical communications theory. But in other contexts, truth-value may not be the only criterion. One application where informational value can be important is with automatic pattern recognition, where not all patterns are of equal value to the agent. For example, consider a photo-intelligence system, where the agent wishes to assign higher informational value to a threatening target than to a benign one, even though the credal probability of a threat may be small. The resulting decision rule would then be very reluctant to reject the hypothesis of a threat without compelling evidence to do so. Considerations of probability of error should never be ignored, but neither should considerations of importance of error.

The introduction of a concept of informational value for each hypothesis, as characterized by the $M$-function, provides a convenient mechanism for the agent to incorporate his values and goals into the decision problem. Expert knowledge, superior explanatory power, simplicity, and ad hoc preference are all legitimate sources of assessing informational value. Thus, the specification of this function, along with the choice of boldness index, provides the agent with a mechanism to modify the decision criterion to implement other criteria in addition to truth-value.

\section{Convexity}

With many decision problems, the specifications of the informational value-determining probability, $M(\cdot)$, the boldness index, $b$, and the credal probability $Q(\cdot)$ are subject to conflict, uncertainty, and ignorance. Strict Bayesian doctrine requires, for a decision to be obtained, that the agent, even if by fiat, declare all conflicts regarding value to be resolved and all forms of uncertainty and ignorance to be reflected in a single choice of credal probability. By so doing, however, he may be forced to choose arbitrarily between rival assumptions for characterizing the options and the available data. Rational agents cannot be expected to be so decisive in their beliefs and values that they are always willing to provide precise numerical specifications for their utilities and credal probabilities. Circumstances do not always provide a basis for resolving conflicts before making a decision.

We may adduce considerations to support the argument that rational agents may be reluctant to always make numerically definite specifications. The Allais paradox [15] is an example of a decision problem where the axioms of utility 
theory dictate a goal-evaluation procedure that does not conform to the wishes of the agent who establishes the utility function. Also, the Ellsberg paradox [16] is an example of a decision problem where the Bayes decision strategy is inconsistent with the wishes of the agent who is specifying the priors. Wisdom dictates, when the agent is not in possession of numerically definite utilities and credal probabilities, that he should be willing to suspend judgment between all rational decisions that are seriously possible on the basis of the information that is in his possession.

\section{A. Valuational Convexity}

Since changes of scale or zero-level do not change the ordering of preference patterns, two utility functions $u$ and $u^{\prime}$ are equivalent if they are related by a positive linear transformation, i.e., if there exists some $\alpha>0$ and real $\beta$ such that

$$
u^{\prime}=\alpha u+\beta .
$$

Two utility functions are said to be conflicting if (6) does not hold for any permissible values of $\alpha$ and $\beta$. One way to address the existence of conflict of utilities $u$ and $u^{\prime}$ is to form a family of utilities consisting of all convex combinations of $u$ and $u^{\prime}$, i.e., to admit the family of utilities $\left\{u_{\gamma}=\gamma u+(1-\gamma) u^{\prime}: \gamma \in[0,1]\right\}$ as representing the goals and values of the agent. The parameter, $\gamma$, represents $X$ 's compromise between the conflicting valuation systems.

Valuational Convexity: If the set of utility functions used to represent $X$ 's goals and values is a convex set of utility functions, a condition of valuational convexity occurs, i.e., $M \in \mathscr{G}_{X, t}$ and $M^{\prime} \in \mathscr{G}_{X, t}$ implies $\alpha M+(1-\alpha) M^{\prime} \in \mathscr{G}_{X, t}$ for all $\alpha \in[0,1]$. Let $\mathscr{G}_{X, t}$ denote this set of utility functions at time $t$. We say that $\mathscr{G}_{X, t}$ is $X$ 's valuation state, and assume that $\mathscr{G}_{X,} \neq \varnothing$ for any $t$, thus ensuring that $X$ is never completely indifferent to the outcome of the decision problem. We shall say that a condition of valuational uniqueness obtains if, for any two utilities $u \in \mathscr{G}_{X, t}$ and $u^{\prime} \in \mathscr{G}_{X, t}$, there are permissible values of $\alpha$ and $\beta$ such that (6) holds.

The above argument is not intended to be a proof that valuational convexity is the best way to deal with unresolved conflict. But, by admitting any convex combination of rival valuation systems to be a valuation system also, the agent does not rule out any potential resolutions of the conflict. By permitting conflicting values to co-exist, there remains the possibility that future information will permit an ultimate resolution.

\section{B. Credal Convexity}

The credal state of agent $X$ is the set of all credal probabilities that are regarded by $X$ as seriously possible probabilistic descriptions of the inquiry under investigation. Let $\mathscr{B}_{X, t}$ denote the credal state for an agent relative to a given inquiry. Suppose $Q \in \mathscr{B}_{X}$ and $Q^{\prime} \in \mathscr{B}_{X}$, are two possible credal probabilities; $X$ is unable to choose between them, and thereby displays his ignorance. Thus, as with assessments of valuation, there may also be conflict when making credal assessments. One way to address this problem is to relax the requirement for a numerically definite credal probability, and consider all convex combinations of $Q$ and $Q^{\prime}$.
Credal Convexity: If the credal state is closed under all finite convex combinations, then a condition of credal convexity occurs. That is, if $Q \in \mathscr{B}_{X, t}$ and $Q^{\prime} \in \mathscr{B}_{X, t}$, then $Q^{\alpha}=\alpha Q+(1-\alpha) Q^{\prime} \in \mathscr{B}_{X, t}$ for $\alpha \in[0,1]$. The agent's ignorance (lack of knowledge) may be expressed through a convex credal state. If $X$ has sufficient knowledge to warrant the specification of a single probability, then a condition of credal uniqueness exists, and the credal state contains exactly one probability. A strict Bayesian would insist on credal uniqueness. But ignorance regarding the prior distribution is one of the reasons why Bayes theory, though intensively studied, is seldom implemented. Convexity permits the agent to incorporate his ignorance into the decision problem by relaxing the requirement that the prior be numerically definite and, instead, specifying a convex region in the space of logically possible probability functions as the set of probabilities that he will consider as serious possibilities for the "true" prior.

Many scholars have rejected credal uniqueness, claiming that such a requirement does not allow a proper representation of knowledge. Alternative approaches based on the use of upper and lower probability measures have been proposed by Koopman [17], [18], Good [19], and Dempster [20] among others [1, see references therein]; DeRobertis and Hartigan have addressed the problem of inference using intervals of measures [21]. Shafer's development of evidential reasoning based on Dempster's rule of combination of evidence provides an alternative to classical probability theory [4] that has received wide use in knowledge representation, data fusion, and expert systems. Kyburg has shown that Levi's theory includes evidential reasoning as a special case and that Levi's theory imposes weaker logical constraints than does evidential reasoning [6].

We now establish an important property of credal convexity. Suppose the set of prior, or unconditional, probabilities forms a convex set. The following theorem [1] guarantees that the set of conditional probabilities is also convex.

Theorem 1: Let $\mathscr{B}_{X, t}$ be a convex set of unconditional probabilities, and define the set of conditional probabilities of the form

$\mathscr{B}_{X, t}^{c}=\left\{Q_{g}(\cdot)=\frac{Q(\cdot \cap g)}{Q(g)}: g \in \mathscr{A}, Q(g)>0 \forall Q \in \mathscr{B}_{X, t}\right\}$.

Then $\mathscr{B}_{X, t}^{c}$ is convex.

Proof: Let $Q \in \mathscr{B}_{X, t} Q^{\prime} \in \mathscr{B}_{X, t}$, and $\alpha \in[0,1]$. For any $g \in \mathscr{A}$ such that $Q(g)>0$ and $Q^{\prime}(g)>0$, and for any $e \in \mathscr{A} c$, we form the conditional probabilities

$$
Q_{g}(e)=\frac{Q(e \cap g)}{Q(g)}, \quad Q_{g}^{\prime}(e)=\frac{Q^{\prime}(e \cap g)}{Q^{\prime}(g)},
$$

both of which lie in $\mathscr{B}_{X, t}^{c}$. For the convex combination

$$
Q^{\alpha}(e)=\alpha Q(e)+(1-\alpha) Q^{\prime}(e),
$$

the conditional probability

$$
Q_{g}^{\alpha}(e)=\frac{Q^{\alpha}(e \cap g)}{Q^{\alpha}(g)}
$$

also lies in $\mathscr{B}_{X, t}^{c}$. The theorem will be proved if there exists 
$\beta \in[0,1]$ such that

$$
Q_{g}^{\alpha}(e)=\beta Q_{g}(e)+(1-\beta) Q_{g}^{\prime}(e)
$$

holds. Direct substitution shows that $(8)$ holds for

$$
\beta=\frac{\alpha Q(g)}{Q^{\alpha}(g)} .
$$

Since $0 \leqslant \beta \leqslant 1$, the theorem is proved.

The following simple illustration shows how this theorem applies to Bayesian conditioning. Suppose $\boldsymbol{y}$ and $\boldsymbol{w}$ are two discrete random variables. Let the marginal probabilities $\left\{P\left[y=y_{i}\right]\right\}_{i=1}^{m}$ and $\left\{P\left[w=w_{j}\right]\right\}_{j=1}^{n}$ be strictly positive for all $i$ and $j$, but not numerically definite. (i.e., they lie in convex sets). We assume, however, that the conditional probability of $\boldsymbol{y}$ given $\boldsymbol{w}$ is numerically definite for all $i$ and $j$. Then the set of conditional probabilities

$$
P\left[\boldsymbol{w}=w_{j} \mid \boldsymbol{y}=y_{i}\right]=\frac{P\left[\boldsymbol{y}=y_{i} \mid \boldsymbol{w}=w_{j}\right] P\left[\boldsymbol{w}=w_{j}\right]}{\sum_{k=1}^{n} P\left[\boldsymbol{y}=y_{i} \mid \boldsymbol{w}=w_{k}\right] P\left[\boldsymbol{w}=w_{k}\right]}
$$

is a convex set. Thus, convex sets of prior probabilities map, upon conditioning, to convex sets of posterior probabilities.

\section{Admissible Decisions}

The agent is committed to seeking additional error-free knowledge. He has a desire for knowledge, but also has a strong desire to avoid error in the process of modifying his corpus. We adopt the position that there is room between the strict Bayesian doctrine of formulating a decision problem under risk, for which the optimal solution is that of maximizing expected utility, and the contrasting approach of making decisions under uncertainty, for which a favorite strategy is maximin. Levi's decision strategy conforms to strict Bayesian principles when decisions are made under risk, and conforms to maximin when decisions are made under uncertainty. Many decision problems, however, do not fit completely within either of these two special cases. In this section we discuss how to deal with these intermediate situations.

Strict Bayesian decision methodology is based upon assumptions of valuational uniqueness and credal uniqueness. If both of these conditions do not obtain, then Bayesian decision theory cannot be rationally justified, since it would force the agent to choose, in an arbitrary and objectionable way, from among the rival valuational systems and credal probabilities that he considers as seriously possible models for his inquiry. By relaxing the requirement for numerically definite utilities or credal probabilities (or both), it may seem difficult to see how to obtain a useful decision. Levi's rule of expected utility, however, applies as well to cases of valuational and credal convexity as to the case of valuational and convex uniqueness.

In the context of the epistemology discussed in this paper, we present the following definitions [1, ch. 4].

Optimality: For a given utility function $u(\cdot)$ and a given credal probability $Q(\cdot)$ that characterizes the decision problem, invoking Levi's rule of expected utility yields an option that is optimal with respect to expected utility relative to $Q$ and $u$. This option will be a member of the Boolean algebra of elements of the ultimate partition, $U$, and will, in general, consist of more than one element of $U$. If only one element of $U$ survives Levi's rule of expected utility, it will be said to be uniquely optimal with respect to expected utility relative to the given $Q$ and $u$.
Admissibility: An option is said to be admissible if and only if the agent is rationally entitled to choose it from between the available options given his corpus, credal state, and goals. ${ }^{3}$ Under conditions of valuational and credal uniqueness, unique optimality implies admissibility, since the one option surviving Levi's rule will have incorporated all of the agent's knowledge, beliefs, and values (decisions under risk). For all other situations, however, the optimality criterion alone is not sufficient to establish admissibility.

$E$-admissibility: An option is said to be $E$-admissible relative to $\mathscr{B}_{X, t}, \mathscr{K}_{X, t}$, and $\mathscr{G}_{X, t}$ if and only if there is a $Q \in \mathscr{B}_{X, t}$ and a $u \in \mathscr{G}_{X, t}$ such that the option is optimal with respect to expected utility relative to that $Q$-function and $u$-function.

$P$-admissibility: One possible criterion of rational choice is that all $E$-admissible options are admissible. This criterion is not acceptable, however, since there may exist a strongest option that is as weak as or weaker than all $E$-admissible options. Such an option would be one of expressing suspension of judgment between all $E$-admissible options, and is termed a $P$-admissible option. If there is no strongest option as weak as or weaker than all $E$-admissible options, then all $E$-admissible options are deemed to be $P$-admissible options.

$S$-admissibility: We now consider those options that have survived the tests for $P$-admissibility. If more than one option survives, expected utility has failed to decide between them, and we must go beyond considerations of expected utility to resolve the question. Recall that the proximate aim of the decision strategy is to acquire new knowledge while striving to avoid error; this was the motivation for the choice of utility function and for the adoption of $P$-admissibility as criteria that a rational agent might adopt. Once we have taken these principles as far as possible, a tie-breaking mechanism must be defined if it is necessary to settle on a unique decision. A conservative approach consistent with avoiding error is to make decisions based upon the maximin principle, and choose an option that maximizes the minimum utility of all $P$-admissible options. An option is said to be $S$-admissible (or security-admissible) if it is a maximin solution for some $u$-function among the $P$-admissible options. $S$-admissibility is not the only reasonable tie-breaking criterion, but other candidates will lead to larger penalties for deciding incorrectly.

Note that $S$-admissible options may be quite different from regular maximin rules where the maximization and minimization operations take place over the entire ultimate partition of feasible options (decisions under uncertainty). Here, the operation takes place only over $P$-admissible options, i.e., those options that have survived the tests based upon expected utility. $S$-admissibility applies only when expected utility fails to yield an admissible option.

\section{Application}

We present two applications to illustrate the behavior of the decision methodology presented in this paper. The first application, though somewhat artificial, serves to illustrate the operation of Levi's epistemology. The second application provides an alternative approach to an important engineer-

${ }^{3}$ The term, admissibility, as used here is not the same as the standard usage of the term in statistics. A rule is said to be admissible, in statistical usage, if no other rule results in uniformly smaller risk (see, e.g., [22, ch. 2]). 
TABLE I

Multiple Hypothesis Testing Problem

\begin{tabular}{clll}
\hline Option & $Q$-value & \multicolumn{1}{c}{$M$-value } & $Q-0.75 M$ \\
\hline$o_{1}$ & 0.49 & $0.34-0.5 \beta$ & $0.235+0.375 \beta$ \\
$o_{2}$ & $\beta$ & 0.13 & $-0.098+\beta$ \\
$o_{3}$ & $0.50-\beta$ & $0.50+0.5 \beta$ & $-0.125-1.375 \beta$ \\
$o_{4}$ & 0.01 & 0.03 & -0.013 \\
\hline
\end{tabular}

ing problem of associating multiple sources and received signals.

\section{A. Multiple Hypothesis Testing}

An agent has a choice between four options $\left\{o_{1}, \cdots, o_{4}\right\}$ Let $\beta \in(0.01,0.4)$ be a parameter, and suppose the system of credal probabilities ( $Q$-function) and valuations ( $M$-function) are given in Table $\mathrm{I}$. This table also indicates the expected utility of these options for $b=0.75$.

It is easy to see that the credal state $\mathscr{B}_{X, t}$ and the set of utilities $\mathscr{G}_{X, t}$ are both convex. From Levi's rule of expected utility, we determine, for $b=0.75$, that the $E$-admissible sets are:

$$
\begin{aligned}
& \text { for } 0.010<\beta \leqslant 0.091 g_{E_{1}}^{*}=\left\{o_{1}, o_{3}\right\} \\
& \text { for } 0.091<\beta<0.098 g_{E_{2}}^{*}=\left\{o_{1}\right\} \\
& \text { for } 0.098 \leqslant \beta<0.400 g_{E_{3}}^{*}=\left\{o_{1}, o_{2}\right\} .
\end{aligned}
$$

Thus, the set of $E$-admissible options is $\left\{g_{E_{1}}^{*}, g_{E_{2}}^{*}, g_{E_{3}}^{*}\right\}$ There is an option that is as weak as or weaker than all $E$-admissible options for this case, namely, the option $g_{P}^{*}=$ $\left\{o_{1}, o_{2}, o_{3}\right\}$, which becomes the $P$-admissible option. Hence, the only option that is not $P$-admissible is the option $o_{4}$, and this is the only option that is rejected on the basis of expected utility for all permissible $Q$-functions and $M$-functions. All other options are serious possibilities. Note that $o_{1}$ is the option that maximizes expected utility, and corre sponds to the classical Bayesian decision under the criterion of minimizing risk. But the essence of this approach is to not rule out unilaterally any option that is a serious possibility-and therefore cannot be rejected if avoidance (but not necessarily minimization) of error is to be considered.

We may obtain the $S$-admissible option as the maximin option over the $P$-admissible options. To do this, we must evaluate the utility function for accepting and rejecting each $o$, resulting in

$$
g_{S}^{*}=\arg \left\{\min _{o \in g^{*}}\left\{-M_{1}(o)\right\}\right\},
$$

which clearly results in $g_{S}^{*}=o_{2}$. Clearly, $o_{2}$ is $S$-admissible. Note that the global maximin solution for this example is $o_{4}$, but this option was rejected by Levi's rule of expected utility, hence is not $S$-admissible.

The goal of the agent is very conservative; he desires to introduce new knowledge into his corpus carefully, and if he is in error, he wishes to limit the maximum damage that can be done. If considerations of expected utility do not completely resolve the issue, he is forced to resort to methods that do not depend upon his credal state-only upon his system of values. In this situation, a rational procedure is to choose the option for which, for all seriously possible options (i.e., those that are $P$-admissible), the worst possible outcome is as small as possible. For this example, acceptance of $o_{1}$ or $o_{3}$ would have lower utility, if in error, than would erroneous acceptance of $o_{2}$.

\section{B. Multiple Data Association}

Consider a multichannel communications system with $n$ sources, transmitting independent real-valued random variables $\left\{x_{1}, \cdots, x_{n}\right\}$, with corresponding probability density functions $\left\{p_{x_{1}}(\cdot), \cdots, p_{x_{n}}(\cdot)\right\}$. Suppose the receiver collects $n$ independent real-valued random signals $\left\{z_{1}, \cdots, z_{n}\right\}$. We assume that each $z_{i}$ is related to one and only one $\boldsymbol{x}_{i_{j}}$ through the conditional density $p_{z_{i} \mid x_{i}}$, where $\left\{j_{1}, \cdots, j_{n}\right\}$ is an unknown permutation of $\{1, \cdots, n\}$. Suppose we know the conditional densities $p_{z_{i} \mid x_{i}}$ for all $i$ and $j,\left\{z_{1}, \cdots, z_{n}\right\}$ is sampled, and we wish to associate each received signal with the correct source and obtain estimates of $\left\{\boldsymbol{x}_{1}, \cdots, \boldsymbol{x}_{n}\right\}$.

This problem is one of joint association and estimation, with estimation being performed simultaneously with determination of the correct association. It differs from multiple hypothesis decision problems often found in communication applications since, unlike the classical single-user problem of determining which signal was sent, we are interested in a network problem-multiple sources transmitting to multiple receivers. Since $\left\{z_{1}, \cdots, z_{n}\right\}$ are independent, we may recast this problem as $n$ independent single-user problems. A conventional way to attack this problem is to obtain the maximum a posteriori probability (MAP) estimate. We first pursue this approach, and compute the posterior densities of all association pairs, yielding

$$
p_{x_{j} \mid z_{i}}(x \mid z)=\frac{p_{z_{i} \mid x_{j}}(z \mid x) p_{x_{j}}(x)}{\int_{-\infty}^{\infty} p_{z_{i} \mid x_{j}}(z \mid \xi) p_{x_{j}}(\xi) d \xi},
$$

for $i, j=1, \cdots, n$.

For each $i \in\{1, \cdots, n\}$, we wish to obtain the MAP estimate $\hat{x}_{\hat{j_{i}}}$ corresponding to some association index $\hat{j}_{i}$. Thus, given $z_{i}=z$,

$$
\left(\hat{x}_{\hat{j}_{i}}, \hat{j_{i}}\right)=\arg \left\{\max _{x, j} p_{x_{j} \mid z_{i}}(x \mid z)\right\} .
$$

That is, the estimated association index, $\hat{j_{i}}$, is the value of $j$ that generates this maximization, and the estimated value of $\hat{x}_{\hat{j}}$ is the value of $x$ that generates the maximization. This decision/estimation rule is only one of possibly several that could be devised to address the question from a traditional point of view. If the posterior distribution is symmetric and unimodal, then the estimate is the mean of the conditional distribution $p_{x_{j} \mid z_{i}}$ given that the association is correct. If the association is not correct, an interpretation of $\hat{x}_{\hat{j}_{i}}$ is not easily made.

Even though the above estimate lays a legitimate claim to optimality, there are a number of questions that may be raised regarding joint estimation and detection strategies such as this. First, there is no guarantee that the association indices, $\left\{\hat{j_{1}}, \cdots, \hat{j_{n}}\right\}$ will all be distinct, hence there may not be a one-to-one association correspondence between the $\boldsymbol{x}_{j}$ 's and the $z_{i}$ 's. Second, there is no clear way to evaluate the separation, in performance, of the answers; e.g., the closeness of the second-best answer to the best one. Even if one were derived, how one would then determine a threshold to distinguish those estimates that are considered feasible and those that are not is far from clear. Such problems are due to the structure of the decision rule-it is designed to provide one and only one answer, and represents this answer to the agent as the correct one. There is no room for negotiation or equivocation. But negotiation, comparison, and re-evaluation 
are precisely the mechanisms that a human decision maker would like to employ to sort out possible conflict and uncertainty surrounding a decision.

We now revisit this problem from the point of view of epistemic utility theory. We first must specify an information-determining probability to reflect the agent's goals and values concerning the associations that are possible. Although there are many ways to assign value, we will adopt a simple rule and specify that the value of rejecting the association of $x_{j}=x$ and $z_{i}=z$ is proportional to the distance between $x$ and $z$.

Suppose we observe $z_{i}=z$. Define the function

$$
m(x \mid z)= \begin{cases}0 & x<z-D \\ \frac{z-x}{D^{2}} & z-D \leqslant x \leqslant z \\ \frac{x-z}{D^{2}} & z<x \leqslant z+D \\ 0 & z+D<x\end{cases}
$$

where the normalizing quantity $D^{2}$ has been introduced to ensure that $m(\cdot \mid z)$ is a probability density function. The quantity $D$ is some real number taken sufficiently large that the agent feels comfortable in rejecting the association of any $x$ that lies outside the region of serious possibility, $R_{z}=\{x: z-D \leqslant x \leqslant z+D\}$. Thus, we unilaterally decide that we will reject the association of $x$ and $z$ if $x \notin R_{z}$. Let $M(\cdot \mid z)$ be an information-determining probability function such that, for any Borel measurable set $S$ we have

$$
M(S \mid z)=\int_{S \cap R_{z}} m(\xi \mid z) d \xi
$$

Thus, the informational value assigned to any $S$ within this region is determined by the density function $m(\cdot \mid z)$. Let $I_{x}$ be an open interval centered at $x$, and suppose that $I_{x} \subset R_{z}$. Then the information-determining probability of associating $I_{x}$ and $z$ is

$$
M\left(I_{x} \mid z\right)=\int_{I_{x}} m(\xi \mid z) d \xi
$$

For $x \in R_{z}$ and $x^{\prime} \in R_{z}$ with $x \neq x^{\prime}$, the condition

$$
M\left(I_{x} \mid z\right)<M\left(I_{x^{\prime}} \mid z\right)
$$

indicates that the informational value of accepting the association of $x$ with $z$ is greater than the value of accepting the association of $x^{\prime}$ and $z$. Other choices for assigning informational value are possible - the particular valuation system chosen corresponds to a nearest-neighbor criterion. We shall assume valuational uniqueness for this problem.

There is often more knowledge available for specification of the conditional densities, $p_{z_{i} \mid x_{j}}$ than there is for the specification of the unconditional densities, $p_{x}$. Consequently, we shall assume that the conditional densities are numerically definite, but that the unconditional densities form a convex set. Let $\mathscr{B}_{j}$ be the initial credal state of $x_{j}$, i.e., $\mathscr{B}_{j}$ is a convex set of densities

$$
\mathscr{B}_{j}=\left\{p_{x_{j}}^{\omega}: \omega \in \Omega_{j}\right\},
$$

where $\Omega_{j}$ is the index set for elements of $\mathscr{B}_{j}$. Each $p_{x_{j}}^{\omega} \in \mathscr{B}_{j}$ is a seriously possible probabilistic description of $\boldsymbol{x}_{j}$. (It may be simpler, upon fị̂st reading, to assume credal uniqueness; in this case, simply ignore the index $\omega$ in the discussion that follows, since $\Omega_{j}$ becomes a singleton set.) As was discussed in Section V and further elaborated upon in [23], a convex set of prior distributions yields a convex set of posterior distributions. Thus, for each $j$ and $i$, the family of posterior densities governing the association of $\boldsymbol{x}_{j}=x$ and $z_{i}=z$, is given by

$$
\mathscr{B}_{j_{i}}=\left\{p_{x_{j} \mid z_{i}}^{\omega}(x \mid z): \omega \in \Omega_{j}\right\}
$$

where

$$
p_{x_{j} \mid z_{i}}^{\omega}(x \mid z)=\frac{p_{z_{i} \mid x_{j}}(z \mid x) p_{x_{j}}^{\omega}(x)}{\int_{-\infty}^{\infty} p_{z_{i} \mid x_{j}}(z \mid \xi) p_{x_{j}}^{\omega}(\xi) d \xi}
$$

is a convex set of densities. These densities provide a measure of the truth-value of the association of each $x$ with $z$. For each $p_{x_{j} \mid z_{i}}^{\omega}(\cdot \mid z) \in \mathscr{B}_{j i}$, the credal probability that the true state lies in an open interval $I_{x} \in R_{z}$, conditioned on the observational value $z_{i}=z$, is

$$
Q_{i}^{\omega}\left(I_{x} \mid z\right)=\int_{I_{x}} p_{x, \mid z t}^{\omega}(\xi \mid z) d \xi .
$$

For $x \in R_{z}$ and $x^{\prime} \in R_{z}$ with $x \neq x^{\prime}$, the condition

$$
Q_{i}^{\omega}\left(I_{x} \mid z\right)>Q_{i}^{\omega}\left(I_{x^{\prime}} \mid z\right)
$$

indicates that the association of $x$ with $z$ is more credible, or believable, than the association of $x^{\prime}$ and $z$.

The decision rule may be formulated in terms of the information-determining probability density, $m(\cdot \mid z)$ and the family of credal probability density functions $\left\{p_{x_{j} \mid z_{i}}^{\omega}(\cdot \mid z): \omega \in\right.$ $\left.\Omega_{j}, j=1, \cdots, n\right\}$. We shall apply Levi's rule of expected utility to this problem. Let $\bar{x}_{j}^{\omega}$ be the expected value of $\boldsymbol{x}_{j}$ under the unconditional distribution $p_{x_{i}}^{\omega}$, let $\hat{x}_{j_{i}}^{\omega}$ be the corresponding conditional expectation of $\boldsymbol{x}_{j}$ given the observation $z_{i}=z$, and let $I_{\bar{x}_{i}^{\omega}}$ be an open interval centered at $\bar{x}_{j}^{\omega}$ with radius $\rho$. We shall say that $\boldsymbol{x}_{j}$ is associated with $z_{i}=z$ under distribution $p_{x j}^{\omega}$ with boldness $b$ if

$$
\bar{x}_{j}^{\omega} \in R_{z}
$$

and

$$
\lim _{\rho \rightarrow 0}\left[Q_{i}^{\omega}\left(I_{\bar{x}_{j}^{\omega}} \mid z\right)-b M\left(I_{\bar{x}_{j}^{\omega}} \mid z\right)\right] \geqslant 0 .
$$

Since the densities are continuous at $\bar{x}_{j}^{\omega},(16)$ holds if

$$
p_{x_{j} \mid z_{i}}^{\omega}\left(\bar{x}_{j}^{\omega} \mid z\right) \geqslant b m\left(\bar{x}_{j}^{\omega} \mid z\right) \text {. }
$$

Equation (15) serves as a very coarse gate, which determines whether or not an association is even feasible. We may absorb this gate directly into the test by defining the function

$$
m^{*}(x \mid z)=\left\{\begin{array}{ll}
m(x \mid z) & x \in R_{z} \\
\infty & x \notin R_{z}
\end{array} .\right.
$$

The association test may be stated as follows: Accept the association of $\boldsymbol{x}_{j}$ under distribution $p_{x_{j}}^{\omega}$ and $z_{i}=z$ if

$$
p_{x_{j} \mid z_{i}}^{\omega}\left(\bar{x}_{j}^{\omega} \mid z\right) \geqslant b m^{*}\left(\bar{x}_{j}^{\omega} \mid z\right) \text {. }
$$

The test (19) is termed the association likelihood ratio test (ALRT). The content of this test is that we accept the association of $\boldsymbol{x}_{j}$ under distribution $p_{x_{j}}^{\omega}$ and $z_{i}=z$ if the posterior density of $\boldsymbol{x}_{j}$, given $z_{i}=z$, when evaluated at 


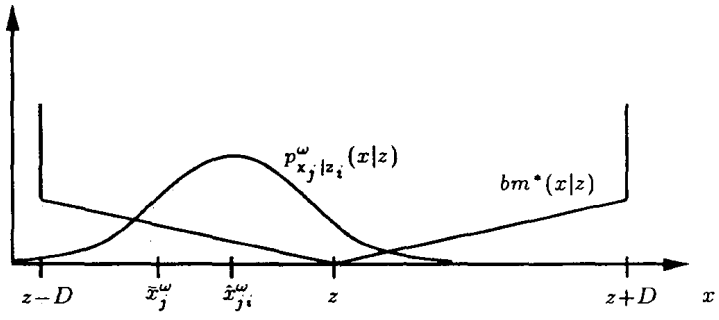

Fig. 1. Association likelihood ratio test.

the mean of the prior density of $\boldsymbol{x}_{j}$, is at least as large as the informational value obtained by rejecting the assiociation of $\boldsymbol{x}_{j}$ and $\boldsymbol{z}_{i}$ (after scaling by $b$ ). Even under conditions of credal uniqueness, however, this decision rule will generally not lead to unique associations, since it is possible that more than one $x_{j}$ will associate with a given $z_{i}$.

Fig. 1 demonstrates, for fixed $\omega$, the informationdetermining and credal probability density functions for this one-dimensional application. Thus, we accept the hypothesis that $\boldsymbol{x}_{j}$ is associated with $z_{i}=z$ since (19) is satisfied for this example.

Credal Uniqueness: We first enumerate the $P$-admissible associations and estimates under conditions of credal uniqueness. Thus, we assume, for each $j \in\{1, \cdots, n\}$, that there is one and only one density function $p_{x_{j}}^{\omega} \equiv p_{x_{j}}$. Let

$$
\hat{J_{i}}=\left\{\hat{j}_{1 i}, \cdots, \hat{j_{k_{i}}}\right\} \subset\{1, \cdots, n\}
$$

denote the indices for which the ALRT is satisfied for $z_{i}=z$, where $k_{i}$ is the number such associations. $\hat{J}_{i}$ corresponds to the $P$-admissible associations for $z_{i}=z$. It is possible that no associations are made, for a given $i$, for any $j \in\{1, \cdots, n\}$. In this case, $k_{i}=0$ and $z_{i}=z$ is said to be dissociated. While this situation may appear to be undesirable, it actually may be quite useful since it allows for the possibility of, say, sensor failure and for the existence of unanticipated and unmodeled signals.

The $P$-admissible estimates are the conditional means of the densities corresponding to $\hat{J}_{i}$, i.e.,

$$
g_{P_{i}}^{*}=\left\{\hat{x}_{k i}: k \in \hat{J}_{i}\right\} .
$$

If there is only one element in $g_{P_{i}}^{*}$, then it is admissible. If $g_{P_{i}}^{*}$ contains multiple entries, then conditions of expected utility have failed to provide a single answer, and a tie-breaking rule must be implemented. An $S$-admissible estimate is one that maximizes the minimum value the epistemic utility can assume. Let $k \in \hat{J}_{i}$. For each interval $I_{\bar{x}_{k}}$ centered at $\bar{x}_{k}$, the utility of erroneously accepting the association is equal, from (4), to $-b M\left(I_{\bar{x}_{k}} \mid z\right)$. The utility per unit length is

$$
\frac{M\left(I_{\bar{x}_{k}} \mid z\right)}{\int_{I_{x_{\star}}} d \xi}
$$

and, as the length of $I_{\bar{x}_{k}} \rightarrow 0$, the utility per unit length converges to $m\left(\bar{x}_{k} \mid z\right)$. The $S$-admissible option is $g_{S_{i}}^{*}=\hat{x}_{k *}$, where

$$
k^{*}=\arg \left\{\min _{k}\left\{m\left(\bar{x}_{k} \mid z\right), k \in \hat{J}_{i}\right\}\right\}
$$

Note, due to the structure of the $M$-function, that the $S$-admissible option corresponds to the nearest-neighbor rule among those association options that have survived Levi's rule of expected utility. The $S$-admissible option is not generally the same as the unconstrained nearest-neighbor decision.

Credal Convexity: Let

$$
\Omega_{j}^{P}=\left\{\omega \in \Omega_{j}: p_{x_{j} \mid z_{i}}^{\omega}\left(\bar{x}_{j}^{\omega} \mid z\right) \geqslant b m^{*}\left(\bar{x}_{j}^{\omega} \mid z\right)\right\} .
$$

If $\Omega_{j}^{P}=\varnothing$, then $x_{j}$ and $z_{i}=z$ are not associated. For each $i$, let

$$
\hat{J}_{i}=\left\{\hat{j}_{1 i}, \cdots, \hat{j}_{k_{i}}\right\} \subset\{1, \cdots, n\}
$$

denote the indices corresponding to non-empty $\Omega_{j}^{P}$. Then the collection of non-empty sets

$$
\hat{\mathscr{T}}_{i}=\left\{\Omega_{k i}^{P}: k \in \hat{J}_{i}\right\}
$$

indexes the collection of all unconditional distributions $p_{x_{k}}^{\omega}$ that are associated with $z_{i}=z$; i.e., the $P$-admissible associations. The corresponding $P$-admissible estimates are

$$
\left.g_{P_{i}}^{*}=\left\{\hat{x}_{k i}^{\omega}: \omega \in \Omega_{k i}^{P}, k \in \hat{J}_{i}\right\}\right\} .
$$

The $S$-admissible option is $g_{S_{i}}^{*}=\hat{x}_{k^{*}}^{\omega *}$, where

$$
\left(k^{*}, \omega^{*}\right)=\arg \left\{\min _{k, \omega}\left\{m\left(\bar{x}_{k} \mid z\right): \omega \in \Omega_{k i}, k \in \hat{J_{i}}\right\}\right\} .
$$

Under credal uniqueness, considerations of expected utility yield a finite number of $P$-admissible numerically definite detection/estimation results, which may be easily evaluated. With credal convexity, however, a continuum of such estimates is obtained. Thus, rather than obtaining a conventional point estimate, we obtain a set estimate. For example, if we adopt the conditional expectation as the estimate, we will obtain a family, or set, of conditional expectations, one for each $\omega \in \Omega_{j}$, each of which is a seriously possible estimate. Point estimation, therefore, is a special case of set estimation under conditions of credal uniqueness. Set-valued estimation has been extended to dynamical systems, and a set-valued Kalman filter has been developed ([24], [23]) that propagates convex sets of conditional expectations (see [25]-[27] for examples).

\section{Discussion}

There are a number of differences between the decision methodology presented in this paper and classical probability-based decision theory.

1) The standard Bayesian cost function is designed to measure the conditional cost of making errors. The incorporation of an unconditional informational value measure (the $M$-function) into the epistemic utility function permits the agent to specify independently a measure of the informational value of each option. While not all applications may benefit from this feature, there may be situations where accounting only for error may not be adequate (e.g., pattern recognition).

2) Decision rules that produce an option that minimizes the cost provide one and only one decision to the agent, which is represented as being the truth. Levi's rule of expected utility provides a decision consisting of all options for which the ratio of credal probability to 
the informational value of rejecting the option exceeds some caution threshold. If a "hard" decision must be made, then some tie-breaking logic must be introduced, such as $S$-admissibility. But many decision problems do not require this rigid decision structure. Application of this approach to sequential decision problems, for example, would permit the agent to retain all seriously possible options until such time that a final decision must be made.

3) Strict Bayesian decision theory requires the unique specification of all utilities and credal probabilities. Valuational and credal convexity, however, relax the requirement for the specification of numerically definite valuation systems and credal probabilities, thus permitting the agent to incorporate a measure of his conflict and ignorance into the decision rule. This capability addresses directly some of the criticisms of strict Bayesian methodology. If a numerically precise prior is not available, a convex family of priors may be defined that does not require more knowledge than is available. The consequence of convexity, however, is that a unique decision or estimate may not be obtainable from considerations only of expected utility.

4) Some of the attractive features of alternatives to probability-based inference may be obtained through convex Bayes decision theory. For example, the DempsterShafer theory of evidential reasoning deals with support and plausibility functions which provide an interval of belief evaluation (see [6] and [4]), rather than a single probability value, but require no fewer assumptions to be made than does the Bayes approach. With the convex Bayes approach, however, it is possible to employ Bayesian conditioning and obtain an interval (a convex set) of belief evaluation.

\section{Conclusion}

When dealing with knowledge representation, there is a temptation to eschew Bayesian decision theory on the grounds that probability-based information processing does not account properly for the representation of ignorance. Convex Bayes conditioning, which permits the employment of convex sets, rather than single, numerically definite, distributions, may alleviate some of the concerns regarding Bayesian inference. By relaxing requirement for the numerical definiteness, however, it may not be possible to obtain unique decisions and point estimates, but the agent is never in a position of asking more from the decision rule than it is possible for it to deliver, given the state of knowledge and ignorance of the agent. Only for situations of valuational and credal uniqueness is it possible, in general, to provide numerically definite results.

Even if probability theory is accepted as the context in which to make inferences, and even if, in addition, numerically definite credal probabilities are available, classical Bayesian decision theory may still not be trusted by the agent if it provides an estimate that is represented to him in a manner that forecloses against another option being seriously considered. In many potential man-machine interface contexts, the agent may wish to be presented with a small number of serious possibilites for final evaluation, rather than to abdicate completely his decision-making authority to an autonomous algorithm. Thus, the application of Levi's rule of expected utility may serve a useful purpose within man-in-the-loop decision-making contexts.

The introduction of informational value into the Bayesian decision-making context seems to originate with Levi. By defining epistemic utility as a compromise between the conflicting goals of avoiding error and acquiring knowledge, a unit of informational value may be apportioned among the options to enable an evaluation of each option on its merits both as to being correct and as to being important. Epistemic utility makes it possible to modify the conventional Bayesian decision-making criterion of minimizing (or limiting) the effect of error to that of seeking error-free knowledge (i.e., of avoiding error). The net result is that, not only should answers be likely to true to warrant the risk of accepting them, but they should be true and informationally valuable.

\section{REFERENCES}

[1] I. Levi, The Enterprise of Knowledge. Cambridge, MA: MIT Press, 1980.

[2] B. van Fraassen, "Rational belief and probability kinematics," Phil. Sci, 47 pp. $165-187,1980$

[3] P. Gärdenfors, Knowledge in Flux. Cambridge, MA: MIT Press, 1988.

[4] G. Shafer, A Mathmatical Theory of Evidence. Princeton, NJ: Princeton Univ. Press, 1976.

[5] L. A. Zadeh, "Fuzzy sets," Inform. Contr., vol. 8, pp. 353-388, 1965.

[6] H. E. Kyburg, Jr., "Bayesian and non-Baysian evidential updating," Artificial Intell.. vol. 31, pp. 271-293, 1987.

[7] D. V. Lindley, "Scoring rules and the inevitability of probability," Int. Statistical Rev., vol. 50, pp. 1-26, 1982.

[8] H. E. Stephanou and A. P. Sage, "Perspectives on imperfect information processing," IEEE Trans. Syst. Man Cybern., vol. SMC-17, pp. $780-798$, Sept /Oct. 1987.

[9] I. Levi, Gambling with Truth. Cambridge, MA: MIT Press, 1967.

[10] __, Decisions and Revisions. London: Cambridge Univ. Press, 1984.

[11] _ , "Imprecision and indeterminancy in probability judgement," Phil. Sci., vol. 52 no. 3, pp. 390-409, 1985.

[12] Hard Choices. London: Cambridge Univ. Press, 1986.

[13] L. A. Zadeh, "Fuzzy Sets," Tech. Rep. Memo. ERL No. 64-44, Univ. Calif., Berkley, 1964.

[14] H. L. Van Trees, Detection, Estimation, and Modulation Theory, Part I. New York: Wiley, 1968.

[15] M. Allais, "Le comportement de l'homme rationnel devant le risque: Critique des postulats et axiomes de l'ecole americaine," Econometrica, vol. 21, pp. 503-546, 1953.

[16] D. Ellsberg, "Risk, ambiguity, and the savage axioms," Economics, 75 pp. 643-669, 1961.

[17] B. O. Koopman, "The bases of probability," Bull. Amer. Math. Soc., vol. 46, pp. 763-764, 1940.

[18] ___, "Intuitive probabilities and sequences," Ann. Math., vol 42, pp. 169-187, 1941.

[19] I. J. Good, "Subjective probability as a measure of a non-measureable set," Logic, Methodology and Philosophy Science, in E. Nagel, P. Suppes, and A. Tarski, editors, Stanford, CA: Stanford Univ. Press, 1962, pp. 319-329.

[20] A. P. Dempster, "Upper and lower probabilities induced by a multi-valued mapping," Ann. Math. Stat., vol. 38, pp. 325-339, 1967.

[21] L. DeRobertis and J. A. Hartigan, "Bayesian inference using intervals of measures," Ann. Statistics, vol. 9, no. 2, pp. 235-244, 1981.

[22] T. S. Ferguson, Mathematical Statistics. New York: Academic Press, 1967.

[23] D. R. Morrell and W. C. Stirling, "Set-valued filtering and smoothing," IEEE Trans. Syst. Man Cybern., 1990, vol. 21, pp. 184-193, Jan./Feb. 1991.

[24] D. R. Morrell, "A theory of set-valued estimation," Ph.D. thesis, Brigham Young University, Provo, UT, 1988. 
[25] D. R. Morrell and W. C. Stirling, "Tracking of Marginally Observable Targets," In Proceedings ICASSP89, pages 2720-2723, May 1989.

[26] W. C. Stirling and D. R. Morrell, "An epistemology of Bayes estimation," in Conf. Rec. Twenty-third Asilomar Conf. Signals, Syst. Comput., Oct. 1989, pp. 699-703.

[27] "A set-valued tracking algorithm using angle-of-arrival data," in Conf. Rec. Twenty-third Asilomar Conf. Signals Syst. Comput., Oct. 1989.

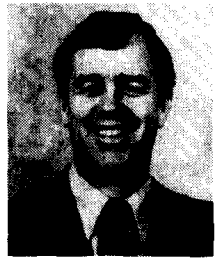

Wynn C. Stirling (M'73) received the Honors B.A. degree magna cum laude in mathematics and the M.S. degree in electrical engineering from the University of Utah, Salt Lake City, in 1969 and 1971, respectively. He received the Ph.D. degree in electrical engineering from Stanford University, Stanford $\mathrm{CA}$, in 1983

From 1972 to 1975 he was with Rockwell International, Anaheim, CA, and from 1975 to 1984 he was employed by ESL, Inc, Sunnyvale, CA. Since 1984, he has been with The Department of Electri- cal and Computer Engineering, Brigham Young University, Provo, UT, where he is an Associate Professor. His current research interests include decision and estimation theory, information theory, and stochastic processes.

Dr. Stirling is a member of Phi Beta Kappa and Tau Beta Pi.

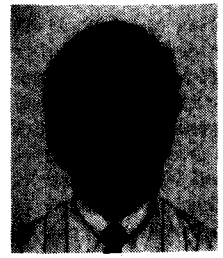

Darryl R. Morrell (S'85-M'88) received the BS, MS, and Ph.D. degrees in electrical engineering from Brigham Young University, Provo, UT, in 1984, 1986, and 1988, respectively.

He is currently an Assistant Professor in the Department of Electrical Engineering Arizona State University, Tempe, AZ. His research interests include statistical decision and estimation theory, stochastic filtering, signal processing, and vector quantization.

Dr. Morrell is a member of Phi Kappa Phi, Tau Beta Pi, and Sigma $\mathrm{Xi}$, and is president of the Phoenix chapter of the Acoustics, Speech, and Signal Processing Society of the IEEE. 